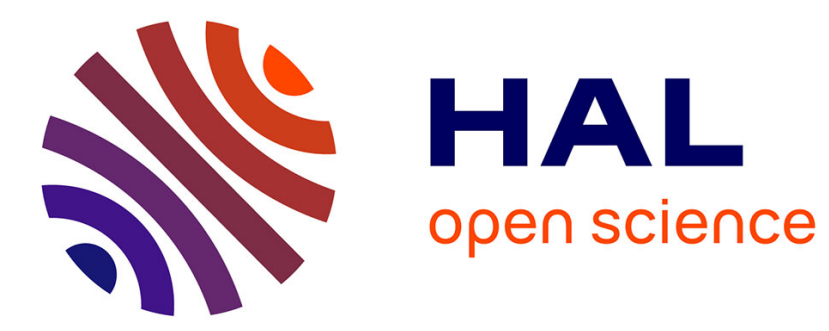

\title{
3D mapping of anisotropic ferroelectric/dielectric composites
}

\author{
Julien Lesseur, Dominique Bernard, U-Chan Chung Seu, Claude Estournès, \\ Mario Maglione, Catherine Elissalde
}

\section{> To cite this version:}

Julien Lesseur, Dominique Bernard, U-Chan Chung Seu, Claude Estournès, Mario Maglione, et al.. 3D mapping of anisotropic ferroelectric/dielectric composites. Journal of the European Ceramic Society, 2015, 35 (1), pp.337-345. 10.1016/j.jeurceramsoc.2014.07.032 . hal-01076000

\section{HAL Id: hal-01076000 https://hal.science/hal-01076000}

Submitted on 16 Jul 2015

HAL is a multi-disciplinary open access archive for the deposit and dissemination of scientific research documents, whether they are published or not. The documents may come from teaching and research institutions in France or abroad, or from public or private research centers.
L'archive ouverte pluridisciplinaire HAL, est destinée au dépôt et à la diffusion de documents scientifiques de niveau recherche, publiés ou non, émanant des établissements d'enseignement et de recherche français ou étrangers, des laboratoires publics ou privés. 


\title{
3D mapping of anisotropic ferroelectric/dielectric composites
}

\author{
J. Lesseur, D. Bernard, U.-C. Chung, C. Estournès, M. Maglione, C. Elissalde
}

\begin{abstract}
Macroscopic anisotropy in polycrystalline materials is of key interest since it may help filling the gap between randomly oriented polycrystals like ceramics and single crystals. Non-destructive X-ray Computed Micro Tomography (XCMT) is a necessary step towards the full control and modelling of such anisotropy, beyond the standard scheme of interfaces. To ascertain this progress, XCMT is applied to 3D mixtures of ferroelectric and dielectric oxides processed by Spark Plasma Sintering (SPS). In such conditions, not only is this anisotropy seen in the overall dielectric parameters but it also shows up in ferroelectric properties. Experimental macroscopic parameters are linked to the 3D morphological anisotropy of individual $\mathrm{MgO}$ inclusions induced during SPS.
\end{abstract}

Keywords: Ceramics; Ferroelectric; Composite materials; 3Dimensional imaging; X-ray computed micro tomography

\section{Introduction}

Three-dimensional composites are finding an increasing numbers of uses since they provide improved functionalities as compared to the simple addition of their individual constituents. Interfaces are usually thought to be involved for such composite effects to appear and their analysis is taken as the first step towards modelling 3D materials. On the other hand, we cannot currently find out the actual shape of the composite phases with the required resolution, with the necessary large-scale extension and in 3-dimensions without destroying the samples. X-ray Computed Micro Tomography (XCMT) meets all these requirements provided that the required resolution is reached. In the field of functional materials, XCMT has been mainly used for fluid flow in porous media, ${ }^{1}$ for the in situ control of sintering ${ }^{2}$ and for mechanical properties. ${ }^{3}$ In the first two cases, the resulting 3D map is the starting point for the implementation of fluid dynamics equations. ${ }^{4}$ In the third, large-scale elastic excitations are usually considered. ${ }^{5}$ This approach is less frequent when materials properties are sensitive to more local fluctuations like dielectrics and this is our main goal here. For this purpose, we processed composites made of a $\mathrm{Ba}_{0.6} \mathrm{Sr}_{0.4} \mathrm{TiO}_{3}$ (BST) ferroelectric matrix in which $\mathrm{MgO}$ inclusions were spread. ${ }^{6-8}$ While the former provides unique dielectric flexibility, the latter drives dielectric losses towards the levels required by the electronic industry. ${ }^{9,10}$ In the present study, we go beyond this accumulation of properties and show a real composite effect. Thanks to the specific conditions of Spark Plasma Sintering (SPS), we are able to produce inclusions with a large aspect ratio (ellipsoid) while preserving the chemical integrity of both phases. As a result of such anisotropic microstructure, overall anisotropy of the dielectric and ferroelectric properties of the BST/MgO composites is induced. We checked that BST alone processed using the same route did not show any such anisotropy. While piezoelectric ceramics with graded porosity have already been reported to show dielectric anisotropy, ${ }^{11,12}$ to the best of our knowledge no ferroelectric anisotropy has been reported. In addition, we propose to link our experimental parameters at low electric field to the 3D morphological anisotropy of the individual $\mathrm{MgO}$ 
inclusions and to the sharp inclusion edges facing each other. This is the first step towards realistic mapping of a possible electric field focusing that would fit with the available empirical models. $^{12-16}$

\section{Experimental}

\subsection{BST and $M g O$ powder mixing}

$\mathrm{Ba}_{0.6} \mathrm{Sr}_{0.4} \mathrm{TiO}_{3}$ nanopowders $(50 \mathrm{~nm})$ were purchased from Pi-Kem (Tilley, UK) (Sections 3.1 and 3.2) and processed by solid-state route at ICMCB (grain size $200 \mathrm{~nm}$ ) (Section 3.3). $\mathrm{MgO}(97 \%)$ supplied by Merck (Darmstadt, Germany) is made of spherical spray-dried granules with an average diameter in the range $10-100 \mathrm{~mm}$. Each granule is composed of packed elementary crystallites of nanometer size. The dry powders were mixed by hand in an agate mortar. To investigate the initial state - i.e. powder bed prior to the sintering step - BST-MgO powder mixtures were introduced inside an SPS tool and consolidated using PMMA as binder.

\subsection{Sintering}

The Spark Plasma Sintering apparatus used was a Dr Sinter SPS-2080 SPS Syntex INC Japan from the Plateforme Nationale CNRS de Frittage Flash (PNF2/CNRS) located in Toulouse (France). Powders (without any sintering aids) were loaded onto a cylindrical die of $8 \mathrm{~mm}$ inner diameter. The temperature was raised to $600{ }^{\circ} \mathrm{C}$ over a period of $3 \mathrm{~min}$, and was then monitored and regulated by an optical pyrometer focussed on a small hole located at the surface of the die. A heating rate of $100^{\circ} \mathrm{C} \mathrm{min}^{-1}$ was used to reach the final temperature of $1200^{\circ} \mathrm{C}$. All the SPS cycles were performed under vacuum and a uniaxial pressure of either $50 \mathrm{MPa}$ or $100 \mathrm{MPa}$ was applied immediately before and until completion of the temperature rising step. The asobtained SPS ceramics were re-oxidized during a post-annealing performed step at $800^{\circ} \mathrm{C}$ for $12 \mathrm{~h}$ in air.

\subsection{Microstructural and dielectric study}

The microstructure of the sintered compacts was studied using a scanning electron microscope JEOL JSM 6360A and a high-resolution scanning electron microscope JEOL 6700F.

Dielectric measurements were performed using a Wayne-Kerr component analyser 6425 for temperatures between $200 \mathrm{~K}$ and $500 \mathrm{~K}$ and in the frequency range: $100 \mathrm{~Hz}-100 \mathrm{kHz}$. The real part of the permittivity derived from the capacitance and dielectric losses $\left(\tan \delta=\varepsilon^{00}{ }_{\mathrm{r}} / \varepsilon_{\mathrm{r}}^{0}\right)$ was directly measured. The piezoelectric resonance was recorded using a HP4194 impedance analyser and the pyroelectric currents were measured with a Keithley 6517B electrometer. The experimental conditions are such that the error bars are estimated smaller than the symbols used in the corresponding figures.

The tunability $(\mathrm{A}(\%)=|\varepsilon(T, E)-\varepsilon(T, E=0)| / \varepsilon(T, E=0) \times 100$, where $E$ is the electric field and $T$ the temperature) was measured by applying a static field of $1 \mathrm{kV} / \mathrm{mm}$ to the sample using a high voltage supply (Bertan Series 225). A homemade decoupling device ensured protection of the impedance meter from the bias. To avoid breakdown in air $(\sim 1000 \mathrm{~V})$, the sample was placed in a silicone oil bath.

\section{4. $3 D$ imaging}

XCMT consists in: first acquiring, for different angular positions, a large number of radiographs of a sample rotating around a fixed axis, and second, in reconstructing from this data set a 3D map of $\mu$, the linear attenuation coefficient of the different components present within the sample. ${ }^{17}$ The coefficient $\mu$ varies with the energy of the X-ray beam and, for each component, it depends on the average atomic number and the density. When the $\mu$ values of the different components are different, 3D $\mu$ mapping can be transformed, using image-processing tools, into a $3 \mathrm{D}$ image of the internal micro-geometry of the scanned sample.

Different X-ray sources can be used for XCMT. In the present work, we used synchrotron radiation from ID19 the 3D X-ray imaging beamline of the ESRF (European Synchrotron Radiation Facility, Grenoble, France) and from TOMCAT the 3D X-ray imaging beamline of the SLS (Swiss Light Source, Villigen, Switzerland). A typical experiment consisted in recording 2000 radiographs $(2048 \times 2048$ pixels $)$ and about 100 references during a continuous rotation through $180^{\circ}$. The energy was equal to $40 \mathrm{keV}$ and the effective voxel size of the reconstructed 3D images equal to $0.52 \mathrm{~mm}$ (ESRF) or $0.37 \mathrm{~mm}$ (SLS).

\section{Results and discussion}

Different parameters can alter the effective properties of multi-materials composed of BST and MgO: the volume fraction of the dielectric phase, the shape of the dielectric inclusions, and the level of chemical inter-diffusion between the two phases. We have already shown that, in ceramics obtained from a mixture of $\mathrm{BST}$ and $\mathrm{MgO}$ powders, increasing the $\mathrm{MgO}$ content up to $10 \mathrm{wt} \%$ within the BST matrix efficiently decreases the dielectric losses but leads to a loss of tunability. The optimal value in order to decrease the low frequency dielectric losses while keeping sufficiently high permittivity tunability is around $4 \mathrm{wt} \% \mathrm{MgO}^{8}$

\subsection{Tuning inclusion shape by pressure}

\subsubsection{Initial microstructure}

First, using SEM we examined the $\mathrm{MgO}$ granules before mixing. They appear as spheroidal particles with a large dispersion in size (Fig. 1a). The mixing process is mild enough to maintain this shape as shown in Fig. 1b where SEM was used to scan a polished section through a sample of the initial mixture consolidated by PMMA impregnation. The section reveals the internal structure of two $\mathrm{MgO}$ particles that appear to be highly heterogeneous, being composed of a several spherical shells. Within the BST matrix some heterogeneities are also visible such as large zones of lower porosity. The cracks that are visible around the $\mathrm{MgO}$ particle and through the matrix are mainly due to the 


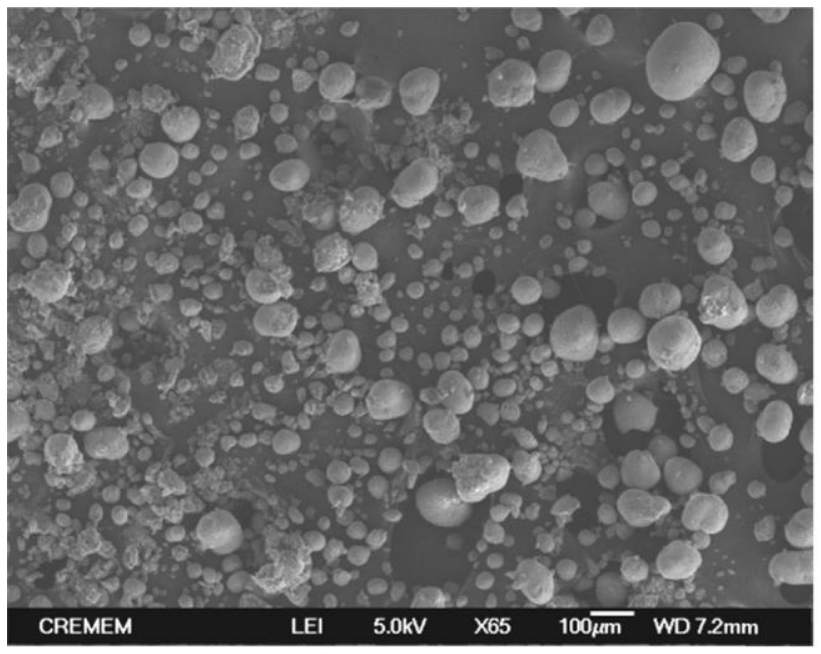

a)

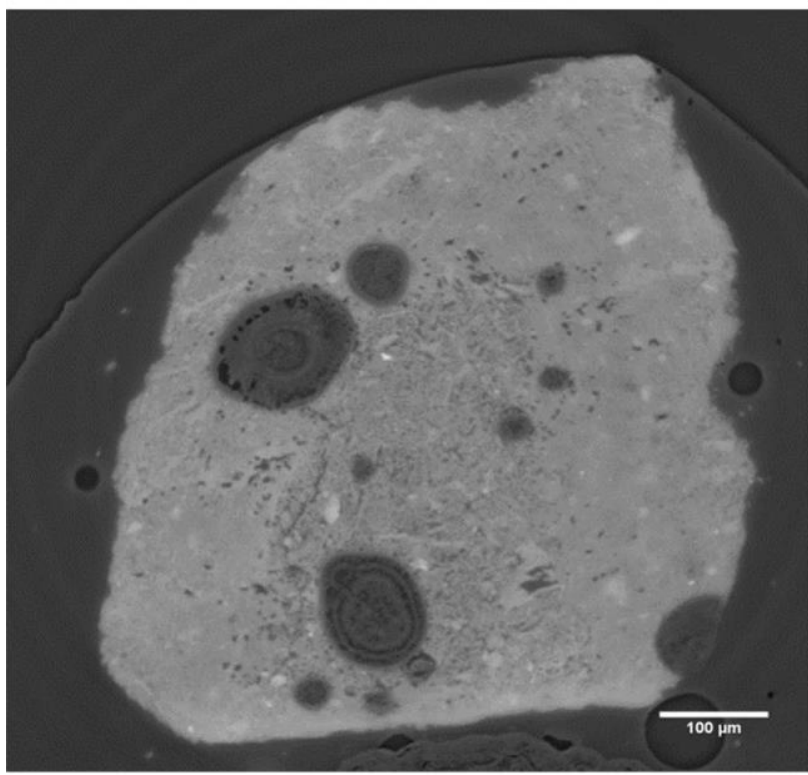

c)

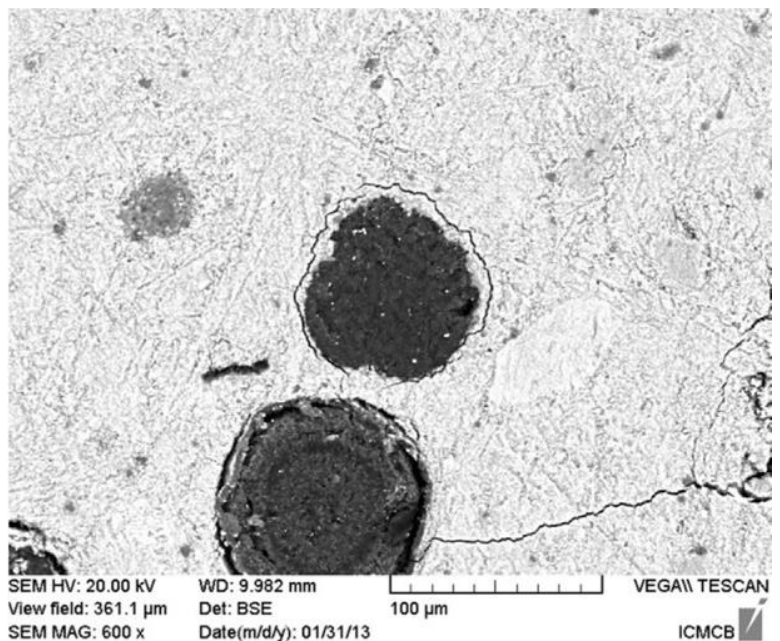

b)

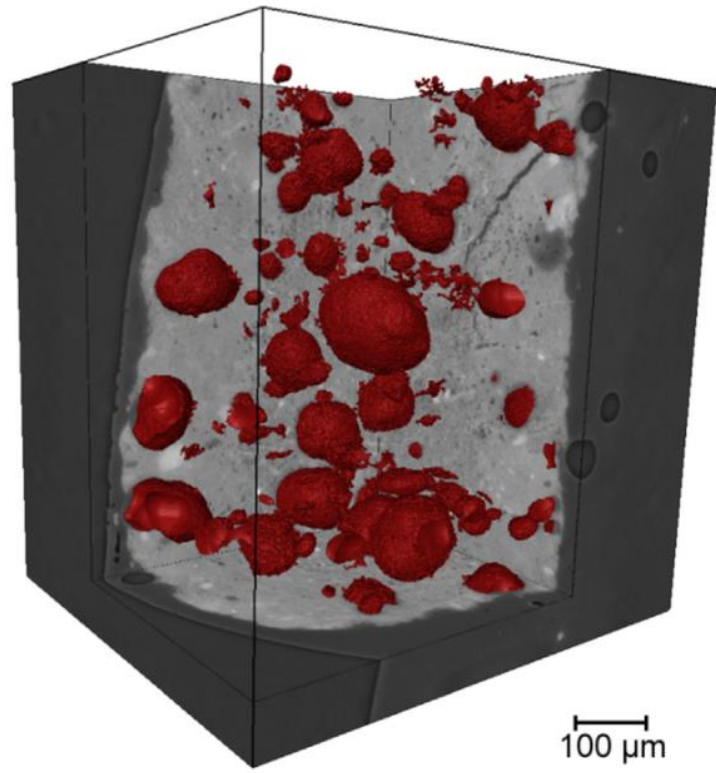

d)

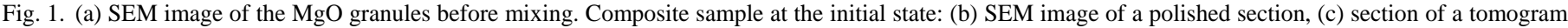
(3D reconstructed image) acquired at ESRF, (d) 3D rendering of the $\mathrm{MgO}$ particles.

sample preparation, impregnation only provides a low mechanical strength to the composite. The observations on the polished section scanned by SEM were confirmed by XCMT (ESRF). The section through the 3D image presented in Fig. 1c presents the same features as in Fig. 1b. The number of cracks is smaller, particularly around $\mathrm{MgO}$ particles, because sample preparation for XCMT is less damaging than for SEM. Of course, having a 3D image of the interior of the sample enables much more complete characterizations than with superficial data only. For instance, the number, the shape, the volume, and several other parameters can be determined for the $\mathrm{MgO}$ particles after a simple segmentation of the 3D image (Fig. 1d).

\subsubsection{Final microstructure}

The use of SPS not only allows densities higher than $95 \%$ to be reached but it also prevents chemical inter-diffusion and cracks at interfaces between the two phases. In addition the pressure applied during SPS is used to flatten the dielectric inclusions. Here we focus on effect of the shape of the inclusions on the dielectric properties. An increase of pressure from 50 to $100 \mathrm{MPa}$ for the composite $\mathrm{BST}-4 \% \mathrm{MgO}$, strongly depresses permittivity in particular in the vicinity of the transition leading to nearly constant values between 200 and $300 \mathrm{~K}$ (Fig. 2). Note that such a pressure increase had no impact on the density that was still higher than $95 \%$. The tunability measured at $100 \mathrm{kHz}$ with an electric field of $1 \mathrm{kV} / \mathrm{mm}$ decreased from $25 \%$ to $18 \%$ upon increasing the pressure from $50 \mathrm{MPa}$ to $100 \mathrm{MPa}$. Globally, the doubling of the pressure decreased permittivity by $40 \%$ whereas the decrease in tunability was only of $7 \%$ (Table 1). We checked whether this modulation of dielectric properties could be related to a change in the composite microstructure and interfaces. The impact of higher pressure on 


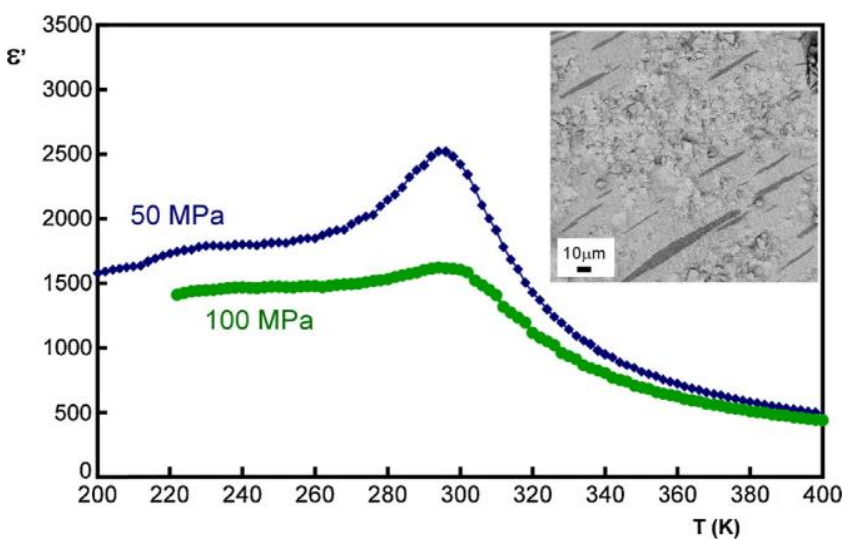

Fig. 2. Thermal variations of dielectric permittivity of BST $+4 \% \mathrm{MgO}$ sintered by SPS at different pressure. Inset: SEM image of fracture surface of the ceramic sintered by SPS a under uniaxial pressure of $100 \mathrm{MPa}$

microstructure results in a narrower grain size distribution of closest BST to the $\mathrm{MgO}$ inclusions $(200-500 \mathrm{~nm})$ and in a slight grain size decrease of the $\mathrm{MgO}$ itself (200-300 $\mathrm{nm}$ ). In addition, thinner and more elongated $\mathrm{MgO}$ inclusions were observed in the composite sintered under $100 \mathrm{MPa}$ pressure (inset Fig. 2). The interfaces were still very clean and without any traces of delamination or cracks. The soft plastic behaviour under SPS of the $\mathrm{MgO}$ nanoparticles constituting the inclusions, and grain rearrangement by sliding could be at the origin of such accommodation that allows tuning of the microstructure anisotropy without degradation of the ceramic. ${ }^{18-20}$ The 3D rendering of the sample (Fig. 3) obtained by XCMT (SLS) clearly reveals that the inclusions appearing as ellipses on the SEM image presented in Fig. 2 are disc-shaped. This is very natural since the only factor that breaks the overall composite symmetry is the uniaxial pressure that is applied during the SPS sintering. In addition, the 3D images confirm that the interfaces between inclusions and matrix are well defined. A small sub-volume can be extracted from the complete sample (black cube in Fig. 3) defining 3D computation domains directly from the 3D geometry. We can thus exclude density, grain size and interfaces as a source of the change in the dielectric parameters. It follows that the increase in the 3D microstructure anisotropy, adjustable by the increase in pressure during the SPS processing is an efficient way to significantly reduce the effective permittivity while maintaining a high tunability. These results are in good agreement with the theoretical approach proposed by Sherman et al. ${ }^{13}$ and demonstrate that the dielectric properties are strongly affected by the

Table 1

Comparison of dielectric permitivity at $T_{\mathrm{c}}$ and room temperature electric field tunability of the permittivity at $100 \mathrm{kHz}$ of BST $+4 \% \mathrm{MgO}$ sintered by SPS at different pressures.

\begin{tabular}{lll}
\hline $\begin{array}{l}\text { SPS BST }-4 \% \\
\mathrm{MgO}\end{array}$ & $\begin{array}{l}\text { Permittivity } \\
\left(T_{\mathrm{c}}-100 \mathrm{KHz}\right)\end{array}$ & $\begin{array}{l}\text { Tunability } \\
(\mathrm{RT}-1 \mathrm{kV} / \mathrm{mm})\end{array}$ \\
\hline $\begin{array}{c}1200^{\circ} \mathrm{C}-3^{0}- \\
P=50 \mathrm{MPa}\end{array}$ & 2500 & $25 \%$ \\
$1200^{\circ} \mathrm{C}-3^{0}-$ & & \\
$P=100 \mathrm{MPa}$ & 1500 & $18 \%$ \\
\hline
\end{tabular}

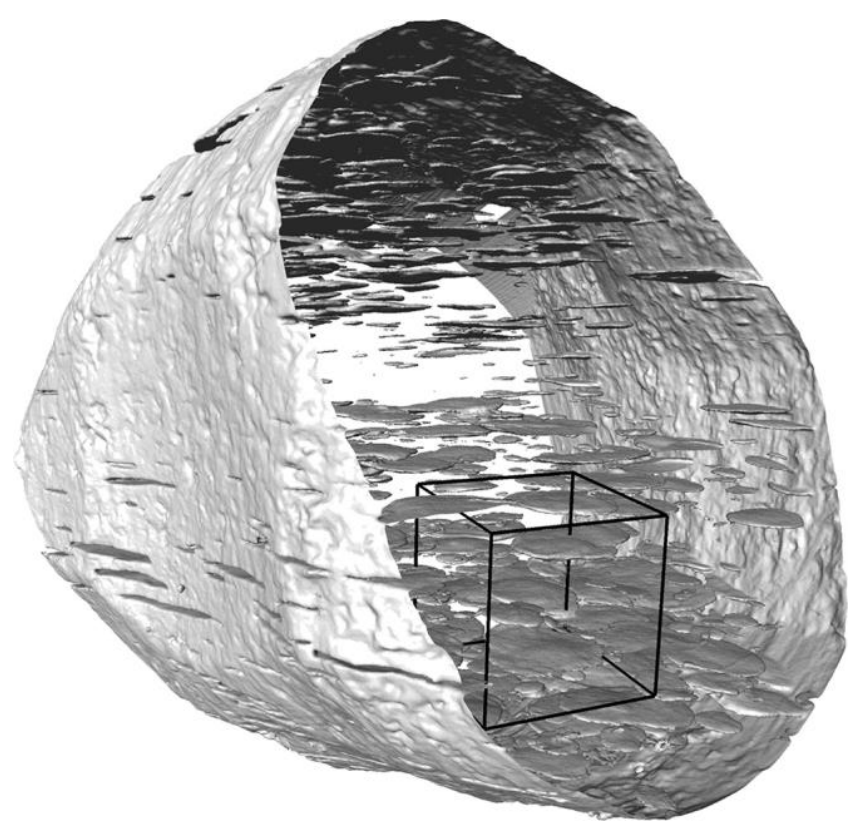

Fig. 3. 3D rendering of a sample in the final state: the external surfaces of the sample and of the dielectric inclusions are visualized. The black cube corresponds to a sub-volume similar to the one used for the permittivity computations.

shape and the distribution of the "passive component" in the ferroelectric matrix that affect the electric field redistribution between the different components. Being able to affect the overall dielectric properties, inclusion anisotropy could also induce anisotropy in the ferroelectric properties of the matrix. This was checked in the next part.

\subsection{Dielectric and ferroelectric anisotropy}

We investigated the dielectric, pyroelectric and piezoelectric properties of the $\mathrm{BST} / \mathrm{MgO}$ composites in two directions relative to the long axis of the $\mathrm{MgO}$ inclusions. In the following, we will use the term parallel to refer to the configuration were the electric field is applied along the inclusions long axis and perpendicular when it is along the thinnest inclusion dimensions. This notation was used for both the small ac electric field, used in dielectric experiments and for the poling electric field used in piezoelectric and pyroelectric experiments. Starting from the pellets that were SPS sintered, parallelepipeds were cut with their major faces parallel either to the pellet surface or to the pellet edges. Samples of BST free from $\mathrm{MgO}$ inclusions were processed in the same way to provide references for all experiments.

The parallel and perpendicular dielectric permittivities are displayed in Fig. 4. The dielectric permittivity is clearly anisotropic, the parallel permittivity being twice the perpendicular one over the whole temperature range. We should first point out that BST samples alone processed in the same way as the $\mathrm{BST} / \mathrm{MgO}$ composites did not display such anisotropy when using exactly the same dielectric set up on samples of similar shape. Since there are no inclusions in pure BST ceramics, the parallel and perpendicular directions were taken relative to the axis of the applied uniaxial pressure during SPS (Fig. 5). We show below that not only the dielectric properties but also the 


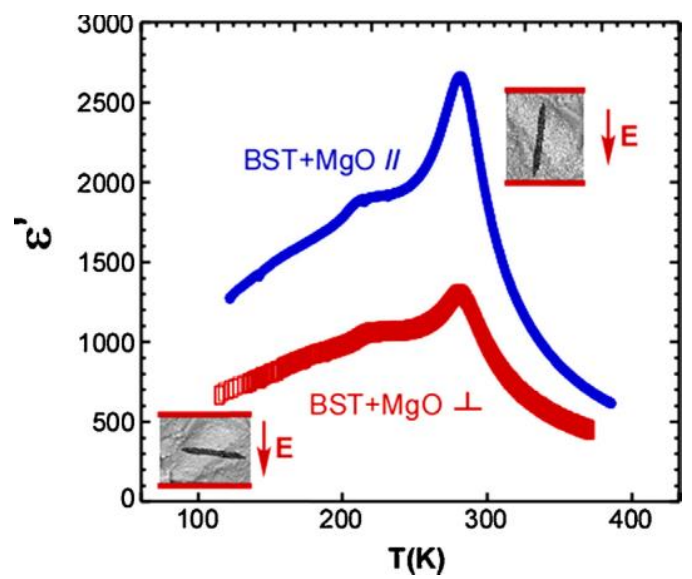

Fig. 4. Permittivity versus temperature of BST $+4 \% \mathrm{MgO}$ SPS ceramics with electrodes parallel and perpendicular to the inclusion long axis.

ferroelectric features are anisotropic in the BST/MgO composites.

To this end, we performed pyroelectric and piezoelectric investigations versus temperature. For the pyroelectric experiments, both perpendicular and parallel samples were poled under the same electric field of $4.7 \mathrm{kV} \mathrm{cm}^{-1}$ on cooling from $350 \mathrm{~K}$ to $100 \mathrm{~K}$. The pyroelectric current was then recorded under heating at a constant rate of $5 \mathrm{~K} / \mathrm{min}$. Integration of this depolarization current and normalization to the sample surface then led to the curves of polarization versus temperature (Fig. 6). The steady state parallel polarization was about 4 -fold $\left(7.8 \mathrm{mC} \mathrm{cm}^{-2}\right)$ the perpendicular one $\left(1.5 \mathrm{mC} \mathrm{cm}^{-2}\right)$. This is strong evidence of a real composite effect since no superimposition of the dielectric properties of the ferroelectric BST matrix on those of $\mathrm{MgO}$ inclusion alter the overall ferroelectric properties of the matrix. This feature was not observed in ceramics that included void inclusions of anisotropic shape. ${ }^{11,12}$ Another probe for ferroelectric anisotropy is the piezoelectric resonance that is less sensitive to all spurious free charges that could always be accumulated at the ferroelectric/dielectric interfaces and that could contribute to the depolarization currents.

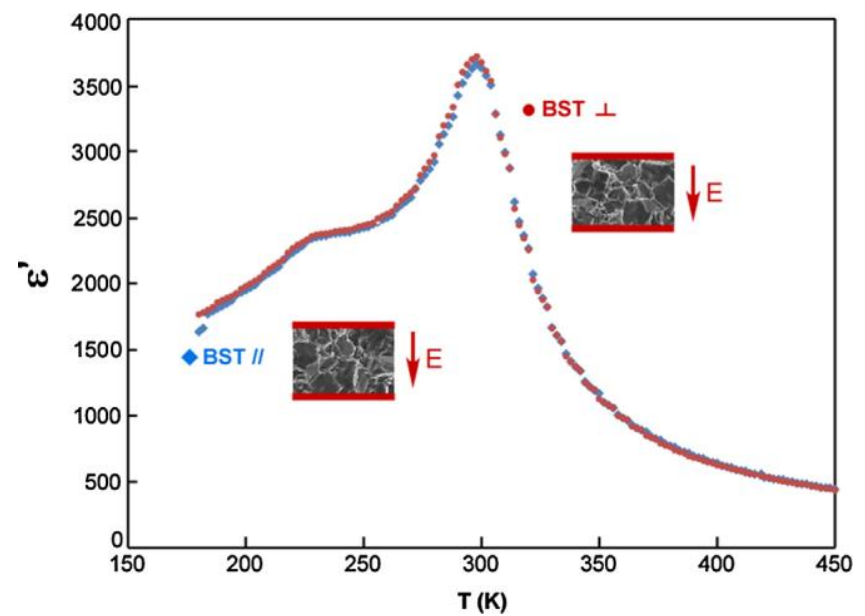

Fig. 5. Permittivity versus temperature of BST SPS ceramics, the notations are the same as for Fig. 4 for the relative orientation of the electric field and the pressure applied during SPS.

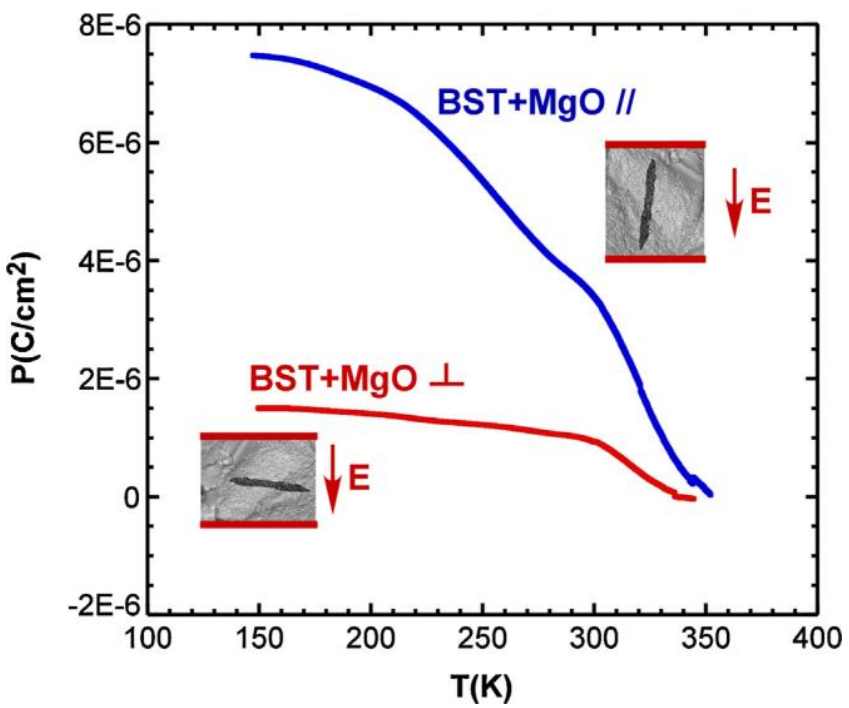

Fig. 6. Polarization versus temperature of $\mathrm{BST}+4 \% \mathrm{MgO}$ SPS ceramics with electrodes parallel and perpendicular to the inclusion long axis.

For the piezoelectric experiments, the parallel sample was a bar $5.59 \times 1.48 \times 0.82 \mathrm{~mm}^{3}$ and the perpendicular sample a Plate $2.28 \times 1.33 \times 0.53 \mathrm{~mm}^{3}$. In both cases, an electric field of $7.3 \mathrm{kV} \mathrm{cm}^{-1}$ was applied on cooling and the piezoelectric resonance was recorded at fixed temperature while heating. We focus on the fundamental piezoelectric resonance mode that is a transverse one using the $\mathrm{d} 31$ piezoelectric coefficient, 3 being the poling direction and 1 the resonating one. Following standard analysis, it is the longest dimension perpendicular to the electric field axis that leads to the $\mathrm{d} 31$ resonance mode. ${ }^{21}$ Fig. 7 gives the resulting piezoelectric resonance seen in conductance in both cases at about $-35^{\circ} \mathrm{C}$. The resonance frequency being exactly inversely proportional to the length of the resonating dimension, one can see in Fig. 6 that the ratio between the resonance frequencies of perpendicular to parallel samples, $f(\perp) / f(q)$, is 2.6

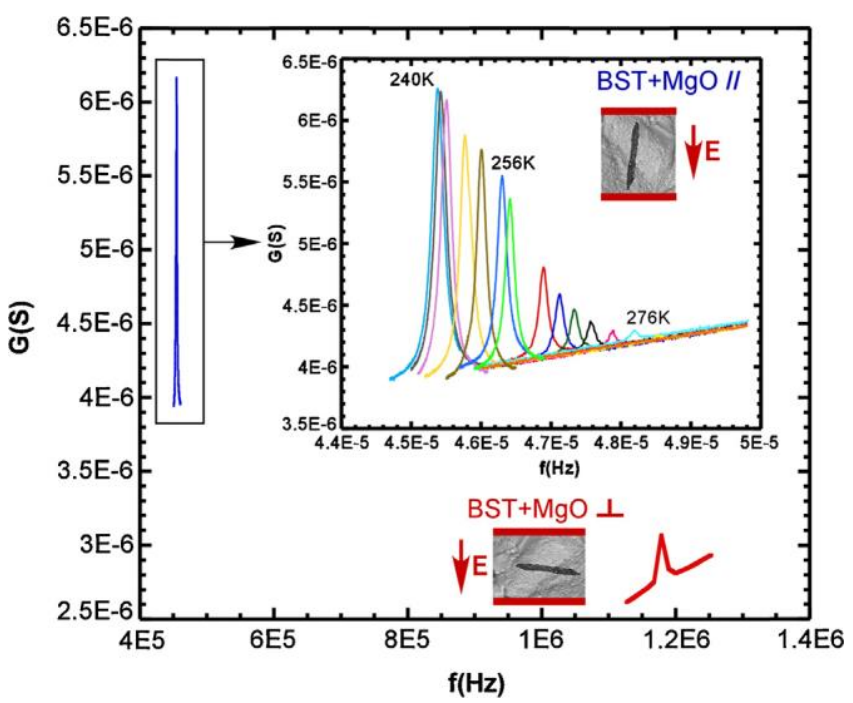

Fig. 7. Conductance versus frequency of ceramics with electrodes parallel and perpendicular to the inclusion long axis. The piezoelectric resonance in the transverse mode is much stronger for the parallel orientation. Inset: temperature dependence of the piezoelectric resonance (parallel configuration). 
while the inverse ratio of lengths, $L(q) / L(\perp)$, is 2.45 . Taking into account the differences in the shape of the two samples, this is a good agreement. What is more striking is that the amplitude of the resonances is at least one order of magnitude smaller in the perpendicular sample than in the parallel one (Fig. 7). Even taking into account the different shapes of the two samples, such a large difference must be due to an intrinsic effect on the piezoelectric coupling coefficients and piezoelectric tensor element $\mathrm{d} 31$. This is in line with the smaller dielectric permittivity and polarization in the former as compared to the latter. Also consistent with the dielectric and pyroelectric data, the piezoelectric resonance disappears at about $5^{\circ} \mathrm{C}$, the transition temperature to the paraelectric non-piezoelectric state.

We can thus summarize this part by stating that not only the overall dielectric properties of the BST/MgO composites are anisotropic, but the pyroelectric and piezoelectric properties of the BST matrix also follow the same trend. It should be noted that the influence of the heterogeneity of the matrix in terms of grain size is not to be discarded. At first sight, it is tempting to ascribe such anisotropy to the anisotropic shape of the $\mathrm{MgO}$ inclusions as evidenced by the SEM pictures shown in Figs. 2 and 3. However, the 2D projection is insufficient to reveal all the features that could lead to ferroelectric anisotropy. In ferroelectric/dielectric composites a number of models have been developed to average out the contributions of the two components of the composites. ${ }^{22-24}$ Sherman et al. ${ }^{13}$ have shown theoretically that dielectric inclusions with sharp edges may lead to large dielectric non-linearities. However, the spatial organization of the phases is three-dimensional and it is of prime importance to take it into account as shown subsequently.

\section{3. $3 D$ properties}

The relation between the ferroelectric properties and the spatial distribution of the $\mathrm{MgO}$ inclusions within the BST matrix is studied using a 3D numerical model able to handle directly the realistic 3D images provided by XCMT.

The equation to be solved is Poisson's equation:

$$
\nabla \cdot[\varepsilon(\mathbf{X}) \nabla V]=0
$$

where $V$ is the potential, $\mathbf{X}$ the $3 \mathrm{D}$ local position and $\varepsilon$ the local permittivity. A permittivity value is attributed to each of the regions identified in the $3 \mathrm{D}$ image.

At the macroscopic scale an external voltage $V_{L}$ is applied to two parallel faces (distance $=L$ ) of a composite sample imposing an average applied electric field $\left(E_{\mathrm{app}}=V_{L} / L\right)$. Assuming that the high-resolution 3D image provided by XCMT is representative of the sample (i.e. all the averaged properties of the sample can be computed using this 3D image), the effective permittivity $\left(\varepsilon_{\text {eff }}\right)$ can be computed resolving Eq. (1) at the microscopic scale (i.e. the scale of the inclusions). For that the problem is reformulated with a new unknown, $w$, defined by the following relation: $V=E_{\text {app }}(w+x)$, where $x$ is the coordinate parallel to the averaged applied field. $E_{\mathrm{app}} w$ is the difference between the local potential and the average one $\left(E_{\mathrm{app}} x\right)$. The problem allowing the computation of $w$ is developed from Eq. (1) (see Annex).
Using a finite volume scheme for the spatial discretization, the effective permittivity in the different directions can be computed for any sample from a representative 3D image (Fig. 3).

To evaluate the relevance of this modelling approach, the experimental results presented in Fig. 8 are compared with numerical results. From the $3 \mathrm{D}$ volume of $1800 \times 1800 \times 1800$ voxels reconstructed from the data acquired at SLS with a pixel size of $0.37 \mathrm{~mm}$, a sub volume of $300 \times 300 \times 300$ voxels $\left(111 \times 111 \times 111 \mathrm{~mm}^{3}\right)$ is extracted after checking its representativeness (same volume fractions that the complete 3D image). The micro-geometry is described with the initial resolution $(0.37 \mathrm{~mm} / \mathrm{pixel})$ and the resulting numerical problem is much smaller, but nonetheless representative.

For this sample the BST matrix is very homogeneous with micrometric grain size (Fig. 8a) and its permittivity is constant at a given temperature. The $\mathrm{MgO}$ permittivity being constant in the temperature range considered here, the effective permittivity of the composite only depends upon two parameters: the local geometry (invariant with temperature) and the matrix permittivity (varying with temperature). The comparison is performed at different temperatures following the subsequent protocol:

1. The numerical effective parallel permittivity is fitted with the experimental value by varying the matrix permittivity. With this step the variability of the BST permittivity with processing conditions is taken into account.

2. The numerical effective perpendicular permittivity is computed using the matrix permittivity determined at step 1 .

A good agreement between the experimental and numerical effective perpendicular permittivity values demonstrates that the numerical model is correctly taking into account the effects of the electrical field distortions induced by the dielectric inclusions (Fig. 8b and c). As shown in Fig. 8, it is effectively the case for the four temperatures tested.

All the computations have been performed in 3D. The effective perpendicular and parallel permittivities are clearly distinct, but parallel permittivity is almost independent from the orientation. Consequently it is reasonable to think that $2 \mathrm{D}$ computation can be sufficient to reveal the effects of the micro geometry on the permittivity values. This supposition has been tested performing $2 \mathrm{D}$ computations on a section of the $3 \mathrm{D}$ volume perpendicular to the inclusions having an $\mathrm{MgO}$ surface fraction equal to the $\mathrm{MgO}$ volume fraction of the 3D image. Parallel permittivity values are in accordance with the 3D results (Fig. 8), but perpendicular permittivity values are systematically lower than the $3 \mathrm{D}$ results, giving a noticeable overestimation of anisotropy. 3D computations are then preferable to $2 \mathrm{D}$ computations from sections if anisotropy of electrical properties is addressed.

Effective Medium Theories (EMT) are widely used to represent the effects on permittivity of inclusions in a matrix. Two very popular laws deduced from these theories are those of Maxwell-Garnett:

$\varepsilon^{e f f}=\varepsilon^{1} \frac{\varepsilon_{1} \varphi_{1}(1-A)+\varepsilon_{2}\left(\varphi_{2}+\varphi_{1} A\right)}{\varepsilon_{1}+A \varphi_{1}\left(\varepsilon_{2}-\varepsilon_{1}\right)}$ 


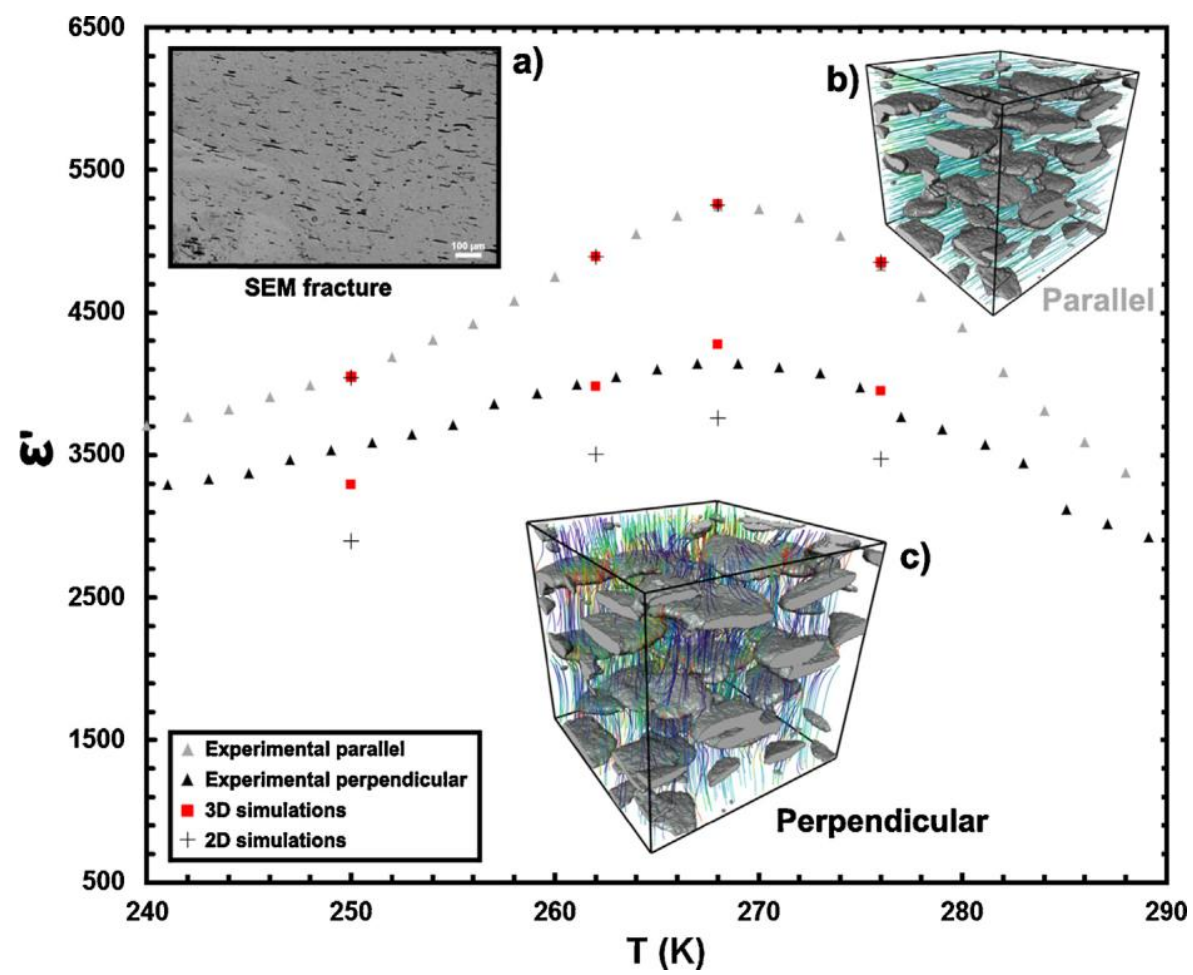

Fig. 8. Permittivity versus temperature of BST (ICMCB powder) $+4 \% \mathrm{MgO}$ SPS ceramics with electrodes parallel and perpendicular to the inclusion long axis. (a) SEM image of a fracture showing the homogeneity of the matrix, (b) and (c) 3D rendering of the inclusions (in grey) and of the computed electrical field.

and Bruggeman:

$\frac{\varepsilon_{e f f}-\varepsilon_{2}}{\varepsilon_{1}-\varepsilon_{2}}-\frac{\varepsilon_{1}}{\varepsilon_{e f f}}=\varphi_{1}$

where $\varepsilon_{\text {eff }}$ is the effective permittivity, $\varepsilon_{1}$ the permittivity of the inclusions with volume fraction $\varphi_{1}, \varepsilon_{2}$ the permittivity of the matrix with volume fraction $\varphi_{2}$, and $A$ the depolarization factor depending on the shape of the inclusions. ${ }^{24}$ To evaluate the applicability of Eqs. (2) and (3), the values of $A$ are estimated from the experimental and numerical results obtained previously (Table 2). Both approaches give a strong contrast between parallel and perpendicular depolarization factors indicating that $A$ is effectively characterizing the anisotropy of the inclusions. Furthermore the obtained values for one equation and one direction ( $\perp$ orq) are remarkably consistent $( \pm 15 \%$ around the average). Predictability has been evaluated using the $A$ values at $268 \mathrm{~K}$ to compute the effective permittivities at the other temperatures. Most of the obtained values are within $1 \%$ of the experimental values ( 8 over 12 ), the others having differences always smaller than $10 \%$.
In spite of the significant qualities of the EMT approaches shown above, 3D numerical modelling can surpass some of their weaknesses:

1. Simultaneous estimates of $\varepsilon_{2}$ and $A$ are not possible with Eqs. (2) and (3) while 3D numerical modelling allows it. This is important when, like in our case, the permittivity of the matrix is not precisely known.

2. Most of the relations produced using EMT are limited to two materials. 3D numerical modelling can potentially handle any number of materials. The composite material, which characterizations are presented in Figs. 2-7, is heterogeneous with a BST matrix comprising zones with large and small grains. As BST permittivity is considerably varying with grain size, ${ }^{14,25}$ the attempts made to fit experimental and numerical results considering a homogeneous matrix totally failed. Encouraging preliminary results have been obtained introducing a new region with a different permittivity around the inclusions, but as XCMT is not sensitive to grain size when the chemical composition is constant, an adapted method has to be developed in order to

Table 2

Estimates of the depolarization factor $A$ appearing in Maxwell-Garnett (subscript MG, Eq. (2)) and Bruggeman (subscript B, Eq. (3)) in the perpendicular ( 1 ) or parallel (q) directions for the four tested temperatures.

\begin{tabular}{|c|c|c|c|c|}
\hline Temperature (K) & 250 & 262 & 268 & 276 \\
\hline$A_{\mathrm{MG}} \perp$ & $4.36 \times 10^{-3}$ & $5.37 \times 10^{-3}$ & $6.11 \times 10^{-3}$ & $6.25 \times 10^{-3}$ \\
\hline$A_{\mathrm{MG}} \mathrm{q}$ & $5.40 \times 10^{-4}$ & $5.65 \times 10^{-4}$ & $5.82 \times 10^{-4}$ & $7.11 \times 10^{-4}$ \\
\hline$A_{\mathrm{B}} \perp$ & $1.67 \times 10^{-1}$ & $2.00 \times 10^{-1}$ & $2.18 \times 10^{-1}$ & $2.15 \times 10^{-1}$ \\
\hline$A_{\mathrm{B}} \mathrm{q}$ & $3.56 \times 10^{-2}$ & $4.25 \times 10^{-2}$ & $4.58 \times 10^{-2}$ & $5.13 \times 10^{-2}$ \\
\hline
\end{tabular}




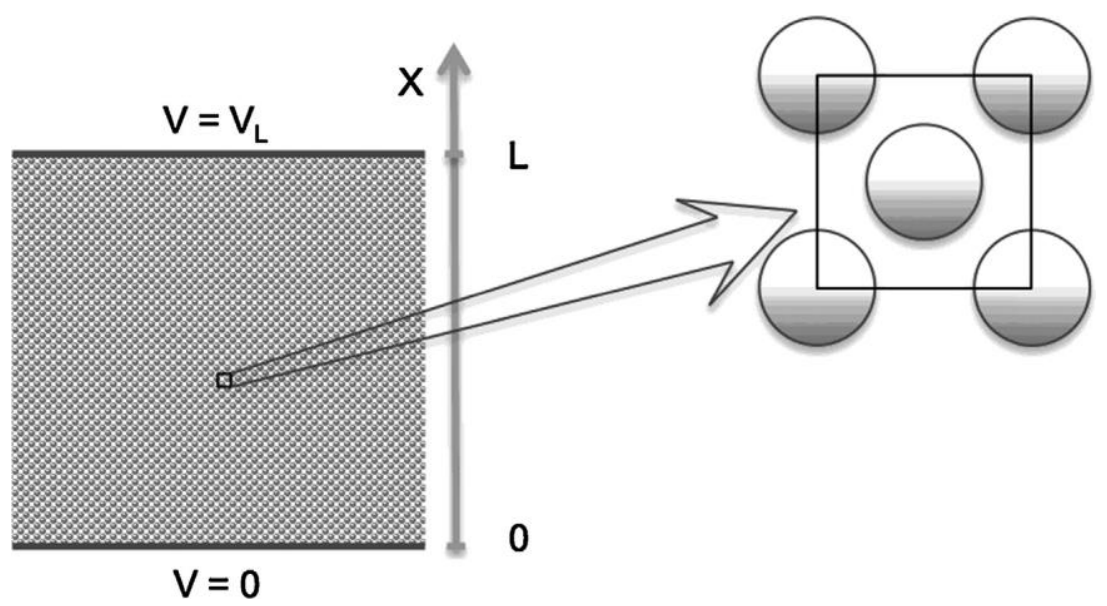

Fig. A1. Diagram of the problem to be solved at the macroscopic scale (left) and the microscopic scale.

objectively delineate the boundary between large and small grains regions.

3. Extension of the 3D numerical approach to non-linear ferroelectric materials is easier than for EMT. Such an extension has been tested and comparisons with experimental tunability data are in progress. Comparisons are complex because one of the critical key points appears to be the non-linear relation used for the permittivity evolution with the local electrical field.

\section{Conclusions}

Thanks to the specific conditions of Spark Plasma Sintering (SPS), anisotropic composites made of dielectric inclusions with a large aspect ratio within a ferroelectric matrix were performed. X-ray Computed Micro Tomography allowed 3D rendering of the sample in both the initial mixed powders and the final ceramic. The initial soft $\mathrm{MgO}$ granules are deformed into flat disc shaped inclusions with sharp edges facing each other. The 3D anisotropy enhanced by the increase of the SPS pressure affects the electric field redistribution between the two phases and appears to be an efficient way to reduce the dielectric permittivity while maintaining electric field tunability. Dielectric measurements performed with electrodes parallel and perpendicular to the inclusion long axis showed overall anisotropy of the dielectric properties of the $\mathrm{BST} / \mathrm{MgO}$ composites. In addition we showed that inclusion anisotropy also induces anisotropy in the piezoelectric and pyroelectric properties of the matrix. To evaluate the effect of the micro geometry on the ferroelectric properties we used a 3D numerical model able to handle the realistic 3D images provided by XCMT directly. XCMT appears to be a relevant tool to link the macroscopic experimental data to 3D morphology in functional multi-materials.

\section{Acknowledgements}

M. Paté and J.P. Ganne from Thales Research \& Technology, rd. 128, 91767 Palaiseau Cedex, France are acknowledged for tunability measurements. This work was supported by the ANR
ARCHIFUN project, grant ANR-12-BS08-009 of the French National Research Agency.

Some of these micro-tomography experiments were performed on the ID19 beamline at the European Synchrotron Radiation Facility (ESRF), Grenoble, France. We are grateful to E. Boller at ESRF for providing assistance in using beamline ID19.

Some of these micro-tomography experiments were performed on the TOMCAT beamline at the Swiss Light Source (SLS), Paul Scherrer Institut, Villigen, Switzerland. We are grateful to R. Mokso at Swiss Light Source whose outstanding efforts made these experiments possible.

\section{Appendix A. Annex}

The objective is to evaluate the effective permittivity of a composite sample simulating the macroscopic experience schematized in Fig. A1. A difference of potential VL is imposed between two parallel opposing faces of a sample. The sample thickness $L$ is very large compared to the size of the inclusions and a direct numerical modelling requires a mesh with small enough elements in order to capture the microstructure revealed by 3D XCMT images. Handling such a large discrete problem is difficult and generally unnecessary. A better approach consists in reformulating the problem at the scale of a representative sub-volume small compared to $L$, but large enough to precisely represent the composite material (Fig. A1).

At this local scale Eq. (1) is still valid, but the boundary conditions are different. The potential $V$ is decomposed into a sum of two terms:

$V=E_{\text {app }}(x+w)$

where $E_{a p p}=V_{L} / L$ is the average applied electric field, $x$ the coordinate perpendicular to the faces at constant potential, and $w$ the new unknown. $E_{a p p} x$ represents the average potential and $E_{a p p} w$ the local deviation of the potential from the average one. Eq. (1) can be reformulated for the new unknown $w$ :

$\nabla \cdot\left[\varepsilon(\mathbf{X})\left(\nabla w+\mathbf{e}_{x}\right)\right]=0$ 
where $\mathbf{e}_{x}$ is the unit vector of direction $\mathbf{X}$. At the internal boundaries between two different regions (noted $i$ and $j$ here) $w$ is continuous and the following boundary condition applies:

$\left(\varepsilon_{i} \nabla w_{i}\right) \cdot \mathbf{n}_{i j}=\left(\varepsilon_{j} \nabla w_{j}\right) \cdot \mathbf{n}_{i j}+\left(\varepsilon_{j}-\varepsilon_{i}\right) \mathbf{e}_{x} \cdot \mathbf{n}_{i j}$

where $\mathbf{n}_{i j}$ is the vector normal to the interface between regions $i$ and $j$ directed from $i$ to $j$.

To close the system boundary conditions must be specified at the external limits. As the computation domain is a Representative Elementary Volume (REV, i.e. a volume which size is smaller than $L$ and larger than the characteristic length of the inclusions, and a volume containing enough inclusions to represent the average behaviour of the material), by analogy with the volume averaging approach ${ }^{26} w$ can be considered as periodic. Periodicity is a weak boundary condition giving the best results when the geometry within the computation domain is not strictly periodic. ${ }^{27} w$ is continuous and the relation (A3) applies when inclusions are cut by the external boundary.

The volume source terms present in Eq. (A2) and the surface source terms present in Eq. (A3) are functions of the permittivity contrasts and of the local geometry. The system (A2) and (A3) is discretized using a classical finite volume scheme and the resulting sparse linear system solved using a parallel solver. The effective permittivity is computed from the heterogeneous $w$ field through the following relation that expresses the conservation of the electrostatic energy of the system:

$$
\mathrm{ZZZ}
$$

$\varepsilon_{\text {eff }}=E_{\text {app }}^{2} \omega=\quad \varepsilon(\mathbf{X}) E^{2} d \omega$

where $\omega$ is the size of the local computation domain and $E$ the local electric field.

\section{References}

1. Bernard D, Nielsen $\varnothing$, Salvo L, Cloetens P. Permeability assessment by 3D interdendritic flow simulations on microtomography mappings of $\mathrm{Al}-\mathrm{Cu}$ alloys. Mater Sci Eng A 2005;392:112-20.

2. Bernard D, Gendron D, Heintz J-M, Bordère S, Etourneau J. First direct 3D visualisation of microstructural evolutions during sintering through $\mathrm{X}$-ray computed microtomography. Acta Mater 2005;53:121-8.

3. Knackstedt M, Arns CH, Saadatfar M, Senden T-J, Limaye A, Sakellariou A, et al. Elastic and transport properties of cellular solids derived from three-dimensional tomographic images. Proc R Soc A 2006;462:2833-62.

4. Flukiger F, Bernard D. A new numerical model for pore scale dissolution of calcite due to $\mathrm{CO}_{2}$ saturated water flow in $3 \mathrm{D}$ realistic geometry: principles and first results. Chem Geol 2009;265:171-80.

5. Limodin N, Réthoré J, Buffière J-Y, Gravouil A, Hild F, Roux S. Crack closure and stress intensity factor measurements in nodular graphite cast iron using three-dimensional correlation of laboratory X-ray microtomography images. Acta Mater 2009;57:4090-101
6. Elissalde C, Estournès C, Maglione M. Tailoring dielectric properties of multilayer composites using spark plasma sintering. J Am Ceram Soc 2007:90:973-6.

7. Chung U-C, Elissalde C, Estournès C, Paté M, Ganne J-P, Maglione M. Low losses, highly tunable $\mathrm{Ba}_{0.6} \mathrm{Sr}_{0.4} \mathrm{TiO}_{3} / \mathrm{MgO}$ composite. Appl Phys Lett 2008;92:0429021-429023

8. Elissalde C, Chung U-C, Artemenko A, Estournès C, Costes R, Paté M, et al. Stoichiometry and grain boundaries control by Spark Plasma Sintering in $\mathrm{Ba}_{0.6} \mathrm{Sr}_{0.4} \mathrm{TiO}_{3}$ : Mn/MgO composites. J Am Ceram Soc 2012;95:3239-45.

9. Sengupta L. Brevet. U.S. 5635434; 1997. Ceramic Ferroelectric Composite material BSTO-Magnesium based Compound.

10. Chang W, Sengupta L. MgO-mixed $\mathrm{Ba}_{0.6} \mathrm{Sr}_{0.4} \mathrm{TiO}_{3}$ bulk ceramics and thin films for tunable applications. J Appl Phys 2002;92:3941-6.

11. Piazza D, Capiani C, Galassi C. Piezoceramic material with anisotropic graded porosity. J Eur Ceram Soc 2005;25:3075-8.

12. Olariu CS, Padurariu L, Stanculescu RE, Baldisserri C, Galassi C, Mitoseriu L. Investigation of low field dielectric properties of anisotropic porous $\mathrm{Pb}(\mathrm{Zr}, \mathrm{Ti}) \mathrm{O}_{3}$ ceramics: experiment and modeling. J Appl Phys 2013;114, 214101/1-9.

13. Sherman VO, Tagantsev AK, Setter N. Model of a low-permittivity and hightunability ferroelectric based composite. Appl Phys Lett 2007;90, 162901/13

14. Curecheriu L, Buscaglia M-T, Buscaglia V, Zhao Z, Mitoseriu L. Grain size effect on the non-linear dielectric properties of barium titanate ceramics. Appl Phys Lett 2010;97, 242909/1-3.

15. Padurariu L, Curecheriu L, Galassi C, Mitoseriu L. Tailoring non-linear dielectric properties by local field engineering in anisotropic porous ferroelectric structures. Appl Phys Lett 2012;100, 252905/1-5.

16. Padurariu L, Curecheriu L, Buscaglia V, Mitoseriu L. Field-dependent permittivity in nanostructured $\mathrm{BaTiO}_{3}$ ceramics: modeling and experimental verification. Phys Rev B 2012;85, 224111/1-9.

17. Buzug T-M. Computed tomography. Berlin, Germany: Springer; 2008.

18. Chaim R, Marder R, Estournès C, Shen Z. Densification and preservation of ceramic nanocrystalline character by spark plasma sintering. Adv Appl Ceram 2012;111:280-5.

19. Crampon J. Creep microstructure of ultrafine-grained $\mathrm{MgO}$ polycristals. Acta Metall 1980;28:123-8.

20. Conrad H, Yang D. Influence of an electric field on the plastic deformation of fine-grained $\mathrm{MgO}$ at high homologous temperatures. Acta Mater 2000;48:4045-52.

21. Mason W-P. Piezoelectric crystals and their application to ultra-sonics. New York: Van Nostrand; 1950.

22. Tuncer E, Serdyuk YV, Gubanski SM. Dielectric mixtures: electrical properties and modeling. IEEE Trans Dielect Elect Insul 2002;9:808-28.

23. Sareni B, Krähenbühl L, Beroual A, Brosseau C. Effective dielectric constant of periodic composite materials. J Appl Phys 1996;80:1688-96.

24. Myroshnychenko V, Brosseau C. Finite-element method for calculation of the effective permittivity of random inhomogeneous media. Phys Rev E 2005;71, 016701/1-16.

25. Frey M-H, Payne DA. Grain-size effect on structure and phase transformations for barium titanate. Phys Rev B 1996;54:3158-68.

26. Whitaker S. The method of volume averaging. Dordrecht: Kluwer Academic Publication; 1999.

27. Kanti T, Forest S, Gallient I, Mounoury V, Jeulin D. Determination of the size of the representative volume element for random composites: statistical and numerical approach. Int J Solids Struct 2003;40:3647-79. 